\title{
Stochastic Modeling and Simulation of Reaction-Diffusion System with Hill Function Dynamics
}

\author{
Minghan Chen \\ Department of Computer \\ Science, Virginia Tech \\ Blacksburg, VA. 24061 \\ cmhshirl@vt.edu
}

\author{
Fei Li \\ Department of Computer \\ Science, Virginia Tech \\ Blacksburg, VA. 24061 \\ felix@cs.vt.edu \\ Yang Cao \\ Department of Computer \\ Science, Virginia Tech \\ Blacksburg, VA. 24061 \\ ycao@cs.vt.edu
}

\author{
Shuo Wang \\ Department of Computer \\ Science, Virginia Tech \\ Blacksburg, VA. 24061 \\ wangshuo@vt.edu
}

\begin{abstract}
In this paper, we demonstrate that in one dimensional domain, highly nonlinear reaction dynamics given by Hill function may have dramatic changes when discretization size is smaller than a critical value. Moreover, we discuss numerical methods to correctly (under certain precision) simulate Hill function dynamics in the microscopic RDME system.
\end{abstract}

\section{Categories and Subject Descriptors}

I.6 [Simulation and Modeling]: Miscellaneous; J.3 [Life and Medical Sciences]: Biology and genetics

\section{General Terms}

Theory

\section{Keywords}

reaction diffusion master equation (RDME), Hill function, stochastic simulation, hybrid method

\section{INTRODUCTION}

Stochastic simulation methods for spatiotemporal biological systems, particularly reaction-diffusion systems can be categorized into two theoretical frameworks: the spatially and temporally continuous Smoluchowski modeling framework [6] and the compartment-based modeling framework, formulated as the spatially discretized reaction-diffusion master equation (RDME) [2]. The Smoluchowski framework stores the exact position of each molecule and is mathematically fundamental, whereas the RDME is coarse-grained and better suited for large scale simulations [1].

Permission to make digital or hard copies of part or all of this work for personal or classroom use is granted without fee provided that copies are not made or distributed for profit or commercial advantage, and that copies bear this notice and the full citation on the first page. Copyrights for third-party components of this work must be honored. For all other uses, contact the owner/author(s). Copyright is held by the author/owner(s).

$B C B^{\prime} 16$, October 2-5, 2016, Seattle, WA, USA.

Copyright 2016 ACM 978-1-4503-4225-4/16/10 ...\$15.00.

http://dx.doi.org/10.1145/2975167.2985668
In RDME, the spatial domain is discretized into small compartments. Within each compartment, molecules are considered "well-stirred". Diffusion is modeled as continuous time random walk on mesh compartments, while the reactions fire only among molecules in the same compartment. It has been proved that the RDME of bi-molecular reactions in 3D domain becomes incorrect and yields unphysical results when the discretization size approaches microscopic scale [4].

\section{HILL FUNCTION DYNAMICS}

We construct a simplified test model of a reaction-diffusion system in one dimension to simplify analysis. In the test model shown in Figure 1, an enzyme species $E$ (typically a transcription factor) is constantly synthesized and degraded. It further up-regulates the DNA expression of a product $P$. The synthesis rate of $P$ is formulated as a Hill function.

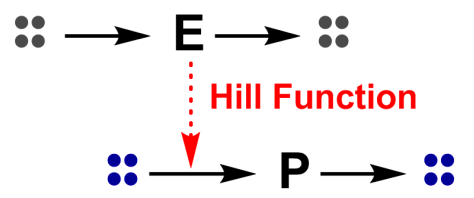

Figure 1: A simple test model of Hill function dynamics.

Assume that a one dimensional (1D) domain of length $L$ is equally partitioned into $K$ compartments with size $h=L / K$ for each. The reaction propensity of the Hill function in compartment $i$ is formulated as $a_{3}=k_{\text {syn }} \cdot h \frac{E_{i}^{4}}{\left(K_{m} \cdot h\right)^{4}+E_{i}^{4}}$.

At the equilibrium state, the distribution of the total population of $E$ is given by the Poisson distribution, $P_{E}(n)=$ $\frac{\alpha^{n}}{n !} e^{-\alpha}$, where $\alpha=\frac{k_{s}}{k_{d}} L$ denotes the mean of the total population of product $P$ in the domain. Then the mean reaction propensity for the synthesis of protein $P$ in the $i$ th bin is

$$
\left\langle a_{\text {syn }}^{i}\right\rangle=k_{\text {syn }} h \sum_{n=0}^{\infty} \frac{n^{4}}{\left(K_{m} \cdot h\right)^{4}+n^{4}} P_{E}^{(i)}(n) .
$$

When the discretization size $h$ is small enough (Hill function approaches one quickly if $n \geq 1$ ), the propensity for the 
product $P$ synthesis reaction can be approximated as

$$
\begin{aligned}
\left\langle a_{\text {syn }}^{(i)}\right\rangle & \approx k_{\text {syn }} \cdot h \cdot \sum_{n=1}^{\infty} P_{E}^{(i)}(n) \\
& =k_{\text {syn }} \cdot h \cdot\left(1-P_{E}^{(i)}(0)\right) \\
& =k_{\text {syn }} \cdot h \cdot\left(1-e^{-\alpha / K}\right) \\
& \approx k_{\text {syn }} \cdot h \cdot \alpha / K
\end{aligned}
$$

Then the total product $P$ population in all $K$ bins is

$$
\langle P\rangle=\frac{k_{\text {syn }}}{k_{\text {deg }}} \cdot \alpha \cdot h .
$$

Equation (3) shows that the total population of product $P$ is a linear function of the mean population of $E$ and the discretization size.

The numerical analysis above are based on two approximations

$$
\left\{\begin{array}{l}
\frac{n^{4}}{\left(K_{m} \cdot h\right)^{4}+n^{4}} \approx 1, \text { for } n \geq 1 \\
e^{-\alpha / K} \approx 1-\alpha / K
\end{array}\right.
$$

Assuming an error tolerance of $5 \%$, the Hill dynamics reduce to a linear function when the discretization bin number

$$
K>\max \left\{2 L K_{m}, \quad 3 \alpha\right\},
$$

\section{FIXED LENGTH SMOOTHING METHOD}

Inspired by the convergent-RDME [5], a remedy for the failure of Hill function dynamics in reaction-diffusion systems is to smooth the population over bins within a certain distance. We choose the smallest bin number $m$ that would not result in failure of the Hill function dynamics, i.e., $m$ such that neither of the two assumptions in the previous analysis are valid. The choice is

$$
m=\left\lceil\max \left\{\frac{0.5}{K_{m} \cdot h}, \frac{0.33 \cdot L}{\alpha \cdot h}\right\}\right\rceil .
$$

Following the terminology in convergent-RDME [5], the "reaction radius $\rho$ " of Hill function dynamics is defined as $\rho=$ $m \cdot h$, where $m$ is given in (6).

Figure 2 shows that the convergent smoothing technique gives very good simulation results for all $h$ values.
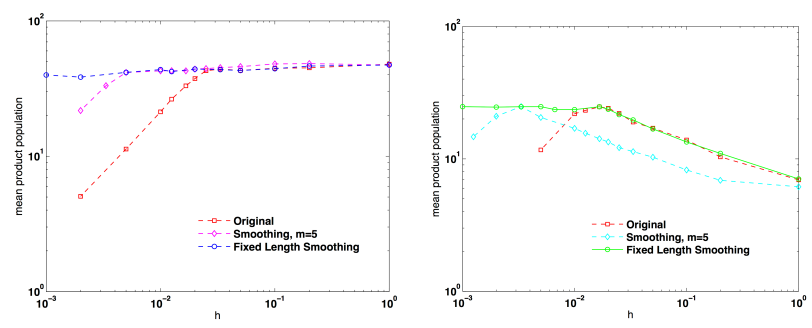

Figure 2: The histogram and the mean population of product $\mathbf{P}$ with different discretization. Parameters: system size $L=1.0, D_{e}=1.0, k_{s}=0.025, k_{d}=0.1, k_{s y n}=0.05$, $k_{\text {deg }}=0.05$. $K_{m}=25.0$ (left), $K_{m}=50.0$ (right).

\section{HYBRID METHOD}

In order to have a stochastic model that is consistent with its deterministic counterpart, the propensity function should take the form $f\left(\left\langle x_{i}\right\rangle\right)$. This motivates us to adopt the hybrid ODE/SSA method and apply it to the reaction diffusion systems. This hybrid method was originally proposed by Haseltine and Rawlings [3].

We applied the hybrid method to the test model. In our simulation, all diffusion events are partitioned into fast systems and solved by ODE solver LSODAR, while the chemical reactions are simulated by SSA under the hybrid framework described above. As an ongoing research, we only tested the case $K_{m}=25$ and Figure 3 shows the corresponding numerical results.
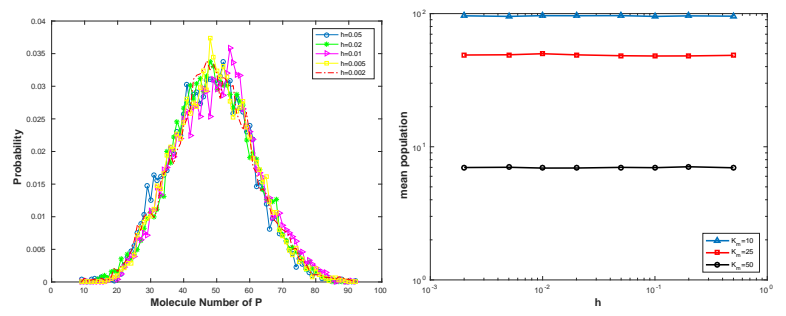

Figure 3: The histogram (left, $K_{m}=25.0$ ) and mean (right) population of product $P$ with different discretization, simulated by the hybrid method. Parameters used here are the same as in Figure 2.

\section{CONCLUSIONS}

An investigation of Hill function dynamics in reactiondiffusion systems reveals that when the discretization size is small enough, the switch-like behavior of Hill function dynamics reduces to a linear function of input signal and discretization size. Proposed fixed reaction radius smoothing method and hybrid method, seems to give a very good remedy to this problem.

\section{ACKNOWLEDGMENTS}

This work was partially supported by the National Science Foundation under awards DMS-1225160, CCF-0953590, and CCF-1526666.

\section{REFERENCES}

[1] D. Fange, O. G. Berg, P. Sjöberg, and J. Elf. Stochastic reaction-diffusion kinetics in the microscopic limit. Proceedings of the National Academy of Sciences, 107(46):19820-19825, 2010.

[2] C. Gardiner, K. McNeil, D. Walls, and I. Matheson. Correlations in stochastic theories of chemical reactions. Journal of Statistical Physics, 14:307-331, 1976.

[3] E. L. Haseltine and J. B. Rawlings. Approximate simulation of coupled fast and slow reactions for stochastic chemical kinetics. The Journal of Chemical Physics, 117(15):6959-6969, 2002.

[4] S. Hellander, A. Hellander, and L. Petzold. Reaction-diffusion master equation in the microscopic limit. Physical Review E, 85:042901, Apr 2012.

[5] S. A. Isaacson. A convergent reaction-diffusion master equation. The Journal of Chemical Physics, 139(5):054101, 2013.

[6] M. von Smoluchowski. Zur kinetischen theorie der brownschen molekularbewegung und der suspensionen. Annalen der Physik, 326(14):756-780, 1906. 